\title{
CERN SPS electron cloud heat load measurements and simulations
}

\author{
V. Baglin and B. Jenninger \\ CERN, 1211 Geneva 23, Switzerland
}

(Received 21 May 2002; revised manuscript received 2 May 2003; published 10 June 2003)

\begin{abstract}
A calorimeter, WAMPAC, operating at room temperature has been designed and installed into the SPS to measure directly the electron cloud induced heat load due to the Large Hadron Collider (LHC)type proton beam. Theoretical behavior, calibrations, measurement protocols, preliminary results, and simulation benchmarking are presented. Scaling of the results to the LHC indicated a linear heating power in a LHC dipole of about $500 \mathrm{~mW} \mathrm{~m}^{-1}$ for $5 \times 10^{10}$ protons/bunch ${ }^{-1}$ for a copper surface which is not fully conditioned (maximum of secondary electron yield $\sim 1.9$ ).
\end{abstract}

DOI: $10.1103 /$ PhysRevSTAB.6.063201

\section{INTRODUCTION}

In the cryogenic elements of the Large Hadron Collider (LHC), the proton beams will be contained inside a perforated "beam screen" (BS), cooled at a temperature between $\sim 5$ and $20 \mathrm{~K}$. Apart from providing pumping, the BS is necessary to intercept the beam induced heat loads such as synchrotron radiation, photoelectrons, and resistive wall losses in order to avoid their dissipation in the $1.9 \mathrm{~K}$ cold bore of the superconducting magnets. Electrons liberated into the beam vacuum chamber are accelerated towards the beam screen due to the electric field of a passing proton bunch. The impact energy of the electrons on the wall produces secondary electrons that may lead to a buildup of an electron cloud due to the successive bunches [1]. Preliminary estimations of the heat load deposited by the electron cloud onto the beam screen indicated a non-negligible contribution to the total heat load budget [1-3]. The last estimations, including elastic reflection of electrons, give linear heat input in the LHC arc dipole of $3.5 \mathrm{~W} \mathrm{~m}^{-1}$ for an unscrubbed copper surface and $0.22 \mathrm{~W} \mathrm{~m}^{-1}$ for a fully scrubbed surface [4]. In the dipole assembly at $\sim 5$ to $20 \mathrm{~K}$ temperature level, the installed cooling power is $1.13 \mathrm{~W} \mathrm{~m}^{-1}$ per aperture [5]. At nominal beam current, the total heat load budget is $0.72 \mathrm{~W} \mathrm{~m}^{-1}$ per aperture. The allocation to the electron cloud is $28 \%$, i.e., $\sim 0.2 \mathrm{~W} \mathrm{~m}^{-1}$ for the dipole field region and $22 \%$, i.e., $\sim 1.9 \mathrm{~W} \mathrm{~m}^{-1}$ for the field free region [6].

An electron cloud activity has been observed in the SPS with LHC-type beams [7]. It is therefore of great importance to measure the heat load deposited by this multipacting effect, in order to benchmark the simulations. For this purpose the WArm MultiPActing Calorimeter (WAMPAC), which measures directly the beam induced heat, was installed at the beginning of 2001 in section 417 , long straight section 4 , of the SPS.

\section{PRINCIPLES}

The calorimeter consists of a thermally floating copper liner, which is installed inside the SPS long straight section type vacuum chamber. This liner is equipped
PACS numbers: 29.20.Dh, 07.20.Fw, 79.20.Hx, 07.30.Kf

with temperature sensors (thermocouple type $E$ ) and a heater for calibration of the calorimeter. The heat load into the calorimeter is measured as a function of the temperature evolution of the liner.

\section{A. Heat equations}

Physically, the heat input to the liner is balanced by the thermal resistance through radiative and contact heat losses and by the warming up of the liner. The dynamic behavior is described with the differential equation below:

$$
\dot{Q}-R \cdot \Delta T-C \Delta \dot{T}=0 .
$$

$\dot{Q}$ is the heat load on the liner, $\Delta T$ is the temperature difference between copper liner $T$ and vacuum chamber $T_{V}, R$ is the thermal resistance between the liner and the vacuum chamber, and $C$ is the thermal capacitance of the liner.

Since initially there is no temperature difference between the copper liner and the vacuum chamber, i.e., $\Delta T(t=0)=0$, and since at equilibrium $\Delta \dot{T}=0$, the solution of the differential equation is

$$
\Delta T(t)=\dot{Q} \cdot R \cdot\left(1-e^{-t / R C}\right) .
$$

With the time constant

$$
\tau=R C,
$$

the slope is

$$
\frac{d \Delta T(t)}{d t}=\frac{\dot{Q}}{C} \cdot e^{-t / R C} .
$$

The thermal resistance $R$ is defined by the two resistances in parallel to the thermal radiation, $R_{\text {rad }}$ and the thermal contact, $R_{\text {cond }}$ :

$$
R=\frac{R_{\mathrm{rad}} R_{\text {cond }}}{R_{\mathrm{rad}}+R_{\text {cond }}} .
$$

For small temperature differences $\Delta T$ between the copper liner and the vacuum chamber, the radiative heat 
flow $\dot{Q}_{R}$ versus the vacuum envelope is

$$
\dot{Q}_{R}=\sigma \varepsilon S F\left(T^{4}-T_{\nu}^{4}\right) \approx \sigma \varepsilon S F 4 T^{3} \Delta T,
$$

where $\sigma=5.67 \times 10^{-8} \mathrm{~W} \mathrm{~m}^{-2} \mathrm{~K}^{-4}$ is the StefanBoltzmann constant, $\varepsilon$ is the effective emissivity, $F$ is the view factor between the liner and the vacuum chamber, and $S$ is the surface area of the copper liner "seen" by the vacuum chamber.

Thus, by definition, the radiative thermal resistance is

$$
R_{\mathrm{rad}}=\frac{\Delta T}{\dot{Q}_{R}} \approx \frac{1}{\sigma \varepsilon S F 4 T^{3}} .
$$

The copper liner is centered inside the vacuum chamber with small stainless steel screws at each end. The conductive resistance between the liner and the vacuum chamber is dominated by the contacts, which makes it difficult to estimate beforehand the conductive thermal resistance by a purely analytical approach. The approach taken was to measure the electrical resistance. The similarity of the mechanisms of thermal and electrical conduction in metals therefore relates the conductive thermal resistance $R_{\text {cond }}$ and electrical resistance $R_{\text {el }}$ [8]. For stainless steel and at room temperature: $\lambda \sim$ $15 \mathrm{~W} \mathrm{~m}^{-1} \mathrm{~K}^{-1}$ (thermal conductivity) and $\rho \sim 7 \times$ $10^{-7} \Omega \mathrm{m}$ (electrical resistivity).

$$
R_{\text {cond }} \approx \frac{R_{\mathrm{el}}}{\lambda \rho} .
$$

The thermal capacitance, $C$, is defined by a specific heat of copper $c$ times the mass of the copper liner $M$ :

$$
C=c M \text {. }
$$

\section{B. Measurements}

The only expected measurable beam induced heat load is due to the electron cloud activity because heat input from image currents are negligible and estimated to be about $5 \mathrm{~mW} \mathrm{~m}^{-1}$ for the nominal LHC beam in SPS (four batches of 72 bunches at $10^{11}$ protons $/$ bunch $^{-1}$ ). Figure 1 shows an ideal measurement cycle for this setup where the relative temperature is plotted versus time. For the analysis of the measurements only temperature changes are taken into account and not the absolute values. When heat is deposited onto the copper liner, the relative temperature increases, following the thermal capacity, up to an equilibrium defined by the thermal resistance. When the heat load is suppressed, the system cools down back to the initial value.

Two independent methods are used to determine the heat load from an ideal measurement cycle:

(i) Using (4) at $t=0$, the measure of the initial warmup slope, which is determined by the thermal capacitance of the copper liner, allows one to compute the heat load.

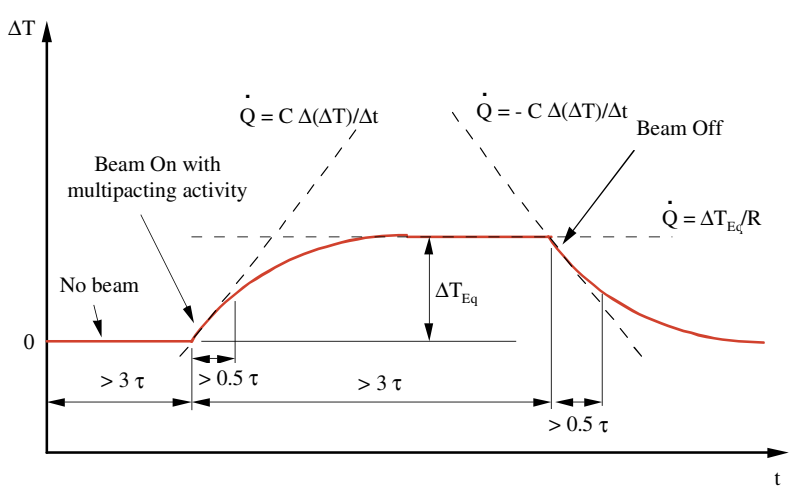

FIG. 1. (Color) Ideal measurement cycle.

To minimize uncertainty in the measurement due to the variation of temperature coming from the beam instability, the slope is measured during the first hour of warming up. In this case, the deviation from the initial warming-up slope, as computed by (4), is $30 \%$ (if the temperature were stable, a slope measured during $5 \mathrm{~min}$ will give a deviation less than 5\%). The start of the cooldown slope from equilibrium is identical to the warm-up slope, but with negative sign [Eq. (1) with the following boundary conditions: $\Delta T(t=0)=\dot{Q} \cdot R$ and $\Delta T(t=\infty)=0$ ].

$$
\dot{Q}=C \frac{\Delta(\Delta T)}{\Delta t}
$$

(ii) Using (2) at $t=\infty$, the measure of the equilibrium temperature $\Delta T_{\mathrm{Eq}}$, which is determined by the thermal conductance to the vacuum envelope, allows one to compute the heat load. In this case, the equilibrium temperature is measured after $3 \mathrm{~h}$ of constant beam condition which gives about $70 \%$ of the correct value.

$$
\dot{Q}=\frac{\Delta T_{\mathrm{Eq}}}{R} .
$$

\section{EXPERIMENTAL SETUP}

\section{A. Description}

Figure 2 shows a schematic of the experimental setup. A circular OFHC copper liner is installed inside an SPS vacuum chamber. This liner is $1.3 \mathrm{~m}$ long, $0.14 \mathrm{~m}$ diameter, and $0.5 \mathrm{~mm}$ thick. The liner has been cleaned according to CERN standard procedure. It is equipped with five, type $E$, thermocouples (TC1, TC2, TC3, TC4, and TC5), which are equally distributed over the length. A calibration heater was brazed over the full length of the liner. Additional thermocouples are installed on the vacuum chamber (TC6) and suspended in the air (TC7) around the experiment. A calibrated Bayard-Alpert vacuum gauge, type 305, and a pickup electrode are installed close to the copper liner to detect the electron cloud activity identified by the pressure rise of the system due 


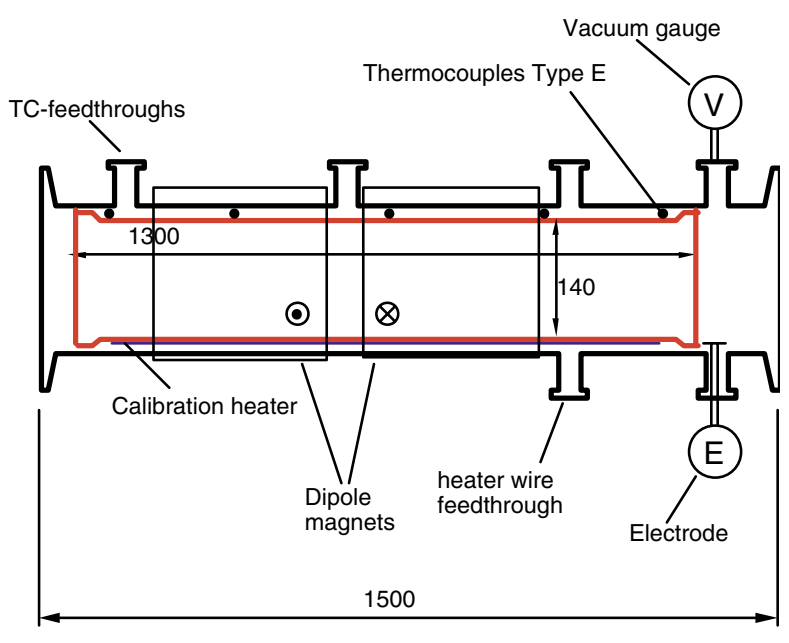

FIG. 2. (Color) Schematic of the WAMPAC calorimeter.

to electron stimulated desorption (ESD). A solenoid coil, wrapped around the vacuum chamber, can be powered to attenuate the multipacting activity. Since the multipacting threshold is lower in a dipole field [9], permanent dipole magnets $(\sim 0.05 \mathrm{~T})$ have been installed over a length of $0.7 \mathrm{~m}$ to trigger multipacting at a lower beam current than in the field free region. Indeed, part of the current limitation in the SPS is due to strong ESD observed in the dipole regions. The data acquisition was performed with a dedicated LabVIEW software. About 100 measurements are averaged and logged every $5 \mathrm{~min}$.

\section{B. Theoretical thermal properties}

The time constant, thermal resistance, and thermal capacitance could be computed and compared with calibration data using Eqs. (3), (5), and (9) and standard data from copper (emissivity, $\varepsilon=0.05$, specific heat of copper, $c=400 \mathrm{~J} \mathrm{~kg}^{-1} \mathrm{~K}^{-1}$ ). The view factor, $F$, of the copper liner inserted into the SPS chamber is assumed to be unity. The copper liner mass, $M$, is $3 \mathrm{~kg}$, has a surface area, $S$, of $0.6 \mathrm{~m}^{2}$, and operates at $T=293 \mathrm{~K}$ (StefanBoltzmann constant $\sigma=5.67 \times 10^{8} \mathrm{~W} \mathrm{~m}^{-2} \mathrm{~K}^{-4}$ ). The measured electrical resistance between the liner and the vacuum chamber was $0.5 \mathrm{~m} \Omega$, corresponding to conductive thermal resistance of $R_{\text {cond }} \sim 48 \mathrm{~K} \mathrm{~W}^{-1}$. The radiative thermal resistance is $R_{\text {rad }} \sim 6 \mathrm{~K} \mathrm{~W}^{-1}$. The total thermal resistance $R$ is therefore dominated by radiation. The corresponding theoretical thermal capacitance, $C$, resistance, $R$, and time constant, $\tau$ are shown in Table I.

TABLE I. Theoretical thermal capacitance, $C$, thermal resistance, $R$, and time constant $\tau$.

\begin{tabular}{ccc}
\hline \hline $\begin{array}{c}C \\
\left(\mathrm{~J} \mathrm{~K}^{-1}\right)\end{array}$ & $\begin{array}{c}R \\
\left(\mathrm{~K} \mathrm{~W}^{-1}\right)\end{array}$ & $\begin{array}{c}\tau \\
\text { (Hours) }\end{array}$ \\
\hline 1200 & 6 & 2 \\
\hline \hline
\end{tabular}

\section{Effect of dipole field on temperature homogeneity}

With the additional dipole field, the heat deposition into the copper liner is not homogeneous. The heat is deposited only along the magnetic fields, i.e., maximum heat deposition at the poles. Longitudinally the heat is mainly deposited in the region with the magnetic field, because of the lower multipacting threshold in the magnetic field region. Therefore, both the thermal diffusion time constants (azimuthal and longitudinal) have to be considered and have to be smaller than the warm-up time constant of the system.

The one dimensional diffusion time constant is related to the thermal diffusivity by (12), the thermal diffusivity being the ratio of the thermal conductivity, $\lambda$, to the product of the material density, $\varphi$, by the specific heat, $c$. For copper $\left(\lambda=400 \mathrm{~W} \mathrm{~m}^{-1} \mathrm{~K}^{-1}\right.$ and $\varphi=$ $8900 \mathrm{~kg} \mathrm{~m}^{-3}$ ), the thermal diffusivity equals $1.1 \times$ $10^{-4} \mathrm{~m}^{2} \mathrm{~s}^{-1}$.

$$
\tau_{D}=\frac{L^{2}}{D}=\frac{L^{2}}{\frac{\lambda}{\varphi c}} .
$$

This diffusion time constant is a measure of the time delay to a change in temperature of a point at the distance $L$ from the heat source. Azimuthally, the distance $L$ is about one-quarter of the tube circumference (i.e., $L_{A} \sim$ $0.11 \mathrm{~m}$ ) and longitudinally it is the length between the end of the magnetic field region and the end of the tube (i.e., $L_{L} \sim 0.3 \mathrm{~m}$ ), therefore, the azimuthal diffusion time constant is $\tau=110 \mathrm{~s}$, and the longitudinal time constant is $\tau=820 \mathrm{~s}$. Thus, both diffusion time constants are small compared to the system time constant, $\tau$, of $2 \mathrm{~h}$.

Similarly, to get a homogenous temperature on the copper liner under steady state conditions, the longitudinal and azimuthal thermal resistances of the liner have to be small compared to the local thermal resistance versus the vacuum envelope.

For a copper liner diameter of $0.14 \mathrm{~m}$ and a thickness of $0.5 \mathrm{~mm}$ and using the azimuthal and longitudinal resistances of the same lengths as for the diffusion time constants, we get the following thermal resistances: azimuthal thermal resistance, $R_{A}=0.9 \mathrm{~K} \mathrm{~W}^{-1}$; longitudinal thermal resistance, $R_{L}=3.4 \mathrm{~K} \mathrm{~W}^{-1}$.

The thermal resistances are still small compared to the resistance versus the vacuum envelope for the same area and do not yet significantly modify the temperature homogeneity. A further reduction of the wall thickness, however, might have a non-negligible influence on the steady state temperature distribution.

\section{Calibration and sensitivity}

The precise values of the thermal capacitance and resistance can be determined during an in situ calibration using the linear heater by applying a known heat load. From Eq. (10), the thermal capacitance is obtained by the 
TABLE II. Measured thermal capacitance, $C$, thermal resistance, $R$, and time constant $\tau$.

\begin{tabular}{ccc}
\hline \hline $\begin{array}{c}C \\
\left(\mathrm{~J} \mathrm{~K}^{-1}\right)\end{array}$ & $\begin{array}{c}R \\
\left(\mathrm{~K} \mathrm{~W}^{-1}\right)\end{array}$ & $\begin{array}{c}\tau \\
\text { (Hours) }\end{array}$ \\
\hline 1330 & 7 & 2.6 \\
\hline \hline
\end{tabular}

initial warm-up slope after switching on the heater. After reaching equilibrium, i.e., a few time constants, the thermal resistance is obtained by Eq. (11). Finally, the time constant, $\tau$, is deduced from Eq. (3). Table II shows the measured thermodynamic properties and demonstrates that the predicted values from Table I agree within $30 \%$ with the measured data.

Figure 3 shows a typical in situ calibration cycle. The increase in the relative temperature, $\Delta(T C i-\mathrm{TC} 6)$ with $i=2,5$, and 7 , is plotted as a function of time when the heater is set to $0.1 \mathrm{~W} \mathrm{~m}^{-1}$. About six calibration measurements were performed in the range 0.02 to $5 \mathrm{~W} / \mathrm{m}$; the average of the measured slopes is $2.7 \mathrm{~K} \mathrm{~W}^{-1} \mathrm{~h}^{-1}$ which corresponds to a thermal capacitance of $1330 \mathrm{~J} \mathrm{~K}^{-1}$. In stable conditions, the apparatus sensitivity is, at least, $0.02 \mathrm{~W} \mathrm{~m}^{-1}$.

\section{RESULTS}

After commissioning of the experimental setup several periods dedicated to electron cloud studies were performed with the SPS. We present here the very first observation of a temperature increase inside the calorimeter.
Figure 4 shows the relative temperature and pressure increases, observed when the LHC-type beam was circulating in the calorimeter. The time axis indicates the number of hours that have passed since recording. At time $<115 \mathrm{~h}$, the SPS was running with standard fixed target beams. During this period the pressure in the system was about $2 \times 10^{-9}$ Torr and only minor temperature variations were observed (TC2, TC3, and TC5), which were mainly due to temperature fluctuations in the SPS tunnel. The machine development (MD) period with the LHC-type beam started at time $=115 \mathrm{~h}$ and lasted until time $=135 \mathrm{~h}$. During this period, several pressure increases up to $10^{-7}$ Torr were observed. These pressure increases are due to ESD from electron multipacting. It should be noted that during this period the other SPS instrumentation devices such as pressure gauges, pickups, strip detectors, etc. also indicated electron cloud activity [10]. In general, the beam conditions were not stable all along this MD period. However, a dedicated period with constant beam parameters over several hours (hour 133-135) could be obtained, enough time to determine the beam induced heat load. This beam was made of three consecutive batches separated by $225 \mathrm{~ns}$ of 72 bunches each, separated by $25 \mathrm{~ns}$ with $\sim 5 \times$ $10^{10}$ protons $/$ bunch $^{-1}$ [10]. During this period a relative temperature increase, close to the sensitivity limit, of about $0.2^{\circ}$ and significant pressure increase is observed. From the measurement of the initial slope during the first hour of the electron cloud activity, a slope of $\sim 0.075^{\circ} \mathrm{h}^{-1}$ could be measured. This slope corresponds to a total deposited power onto the calorimeter of $\sim 30 \mathrm{~mW}$. At time $>135 \mathrm{~h}$, the MD was completed and SPS was

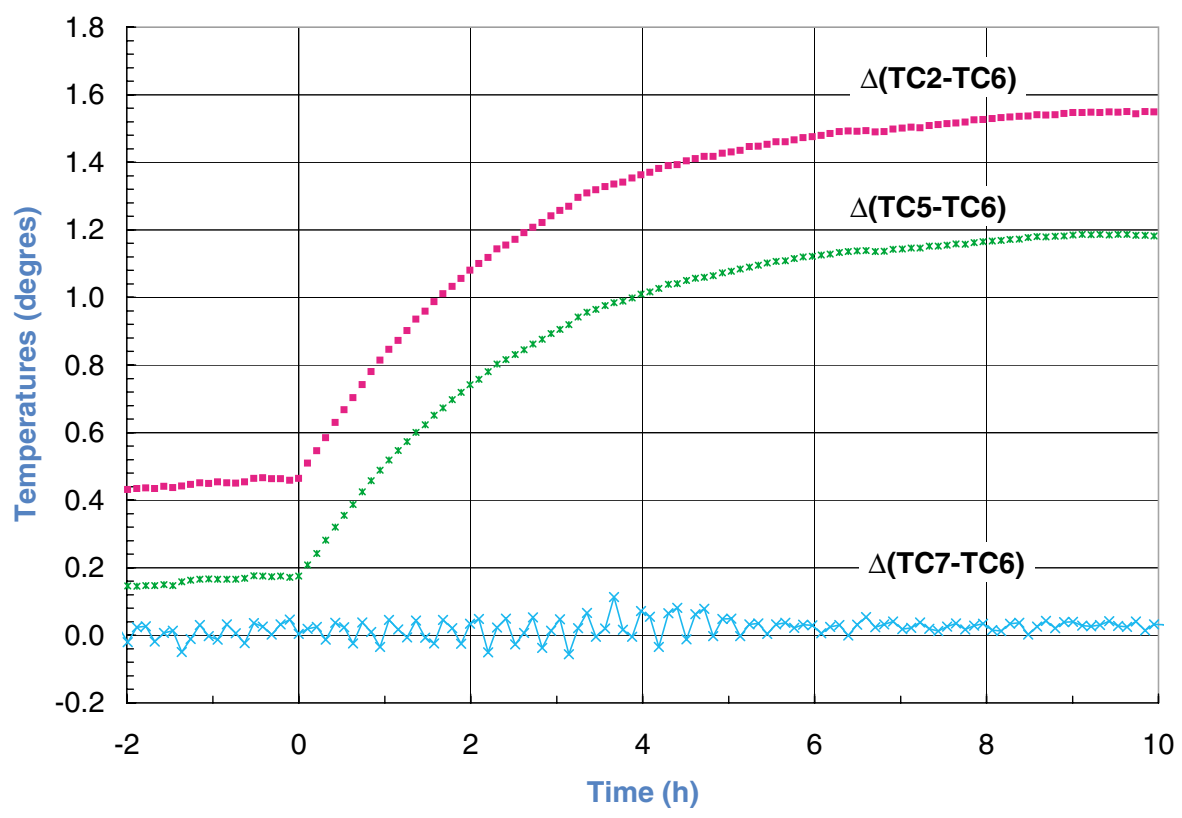

FIG. 3. (Color) Typical in situ calibration cycle. The relative temperature increase corresponds to $0.1 \mathrm{~W} / \mathrm{m}$. The average of the measured slopes is $2.7 \mathrm{~K} \mathrm{~W}^{-1} \mathrm{~h}^{-1}$. 
01-05-03 17:10 \#6_Heatload.xlsanalysed (4)

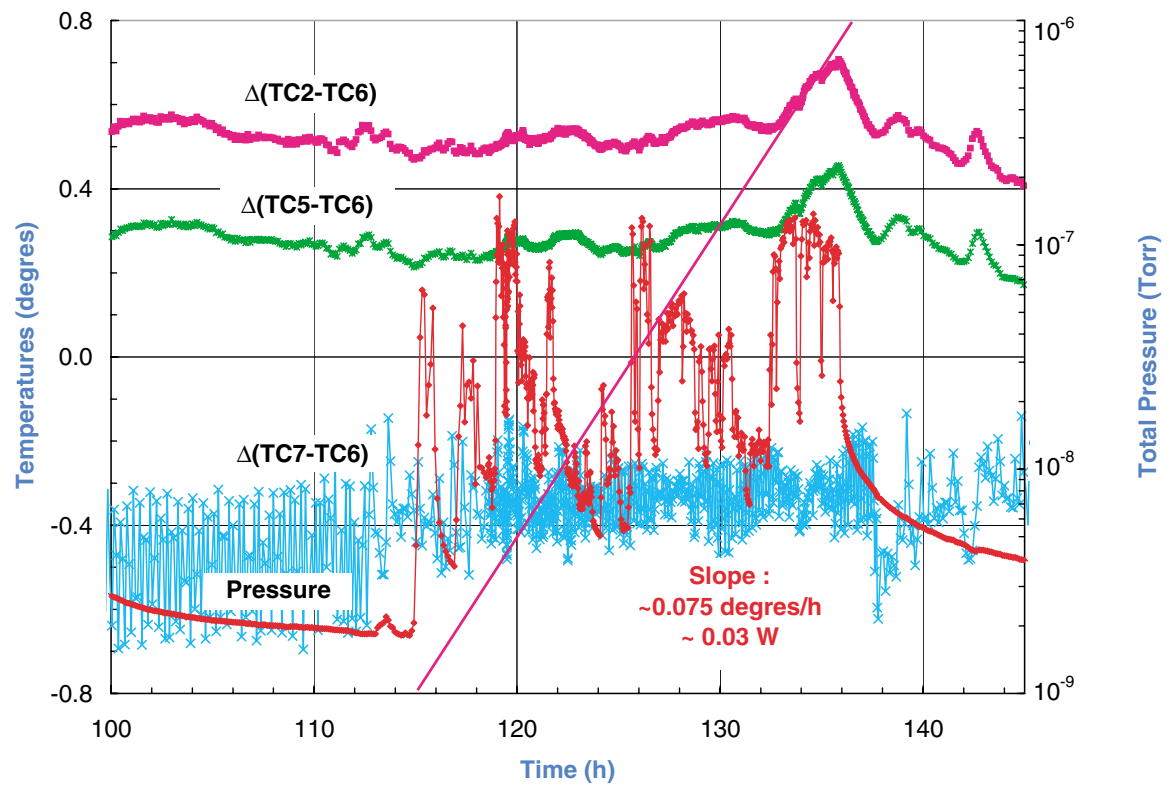

18h19-13h28

V. Baglin and B. Jenninger
LHC/VAC

FIG. 4. (Color) Relative temperature and pressure observations when a LHC-type beam of three consecutive batches of 72 bunches with $5 \times 10^{10}$ protons/bunch ${ }^{-1}$ was circulating in the SPS.

back to normal operation. The relative temperatures and pressure recover to their previous value before MD.

Figure 5 shows the detail of the relative temperature increase observed during the electron cloud activity depicted in Fig. 4. As mentioned in Sec. II B, if the heat input is constant during a time larger than a few time constants, here about 1.5 time constants, the warmup slope is almost equal to the final cooldown slope. The values of the two slopes are in relatively good agreement. The measure of the equilibrium temperature after $3 \mathrm{~h}$ of operation gives a similar heat load as in the slope measurement case, i.e., 40 to $60 \mathrm{~mW} / \mathrm{m}$.

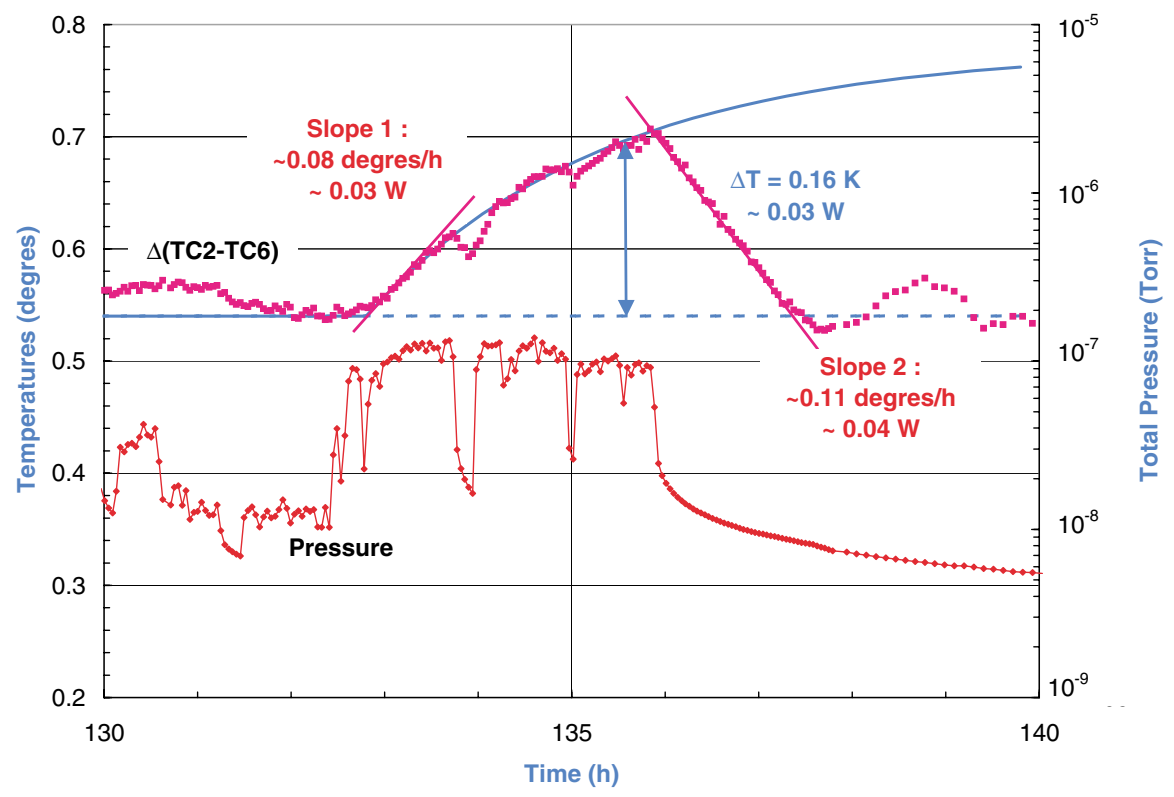

FIG. 5. (Color) Detail of the relative temperature increase observed during electron cloud activity of Fig. 4. 


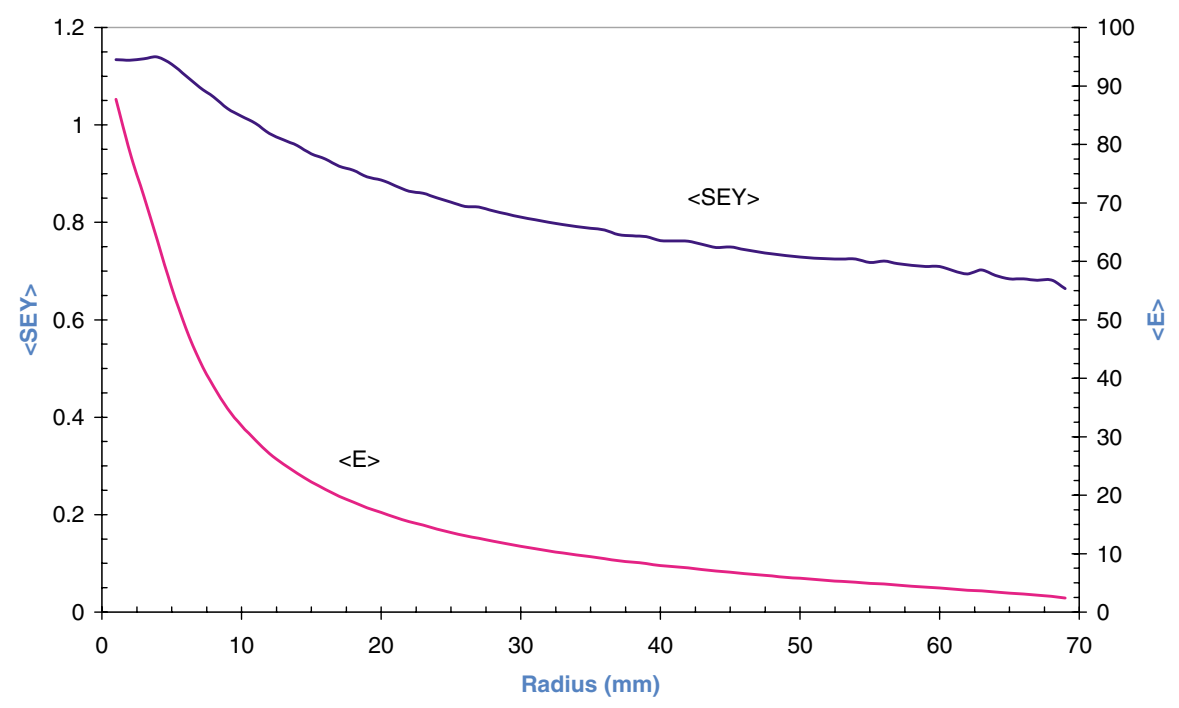

FIG. 6. (Color) Average $\langle\mathrm{SEY}\rangle$ and average electron energy versus the radial position perpendicular to the field lines.

\section{BENCHMARKING SIMULATIONS}

The measurements presented in Sec. IV are used to perform a preliminary benchmarking of two kinds of simulation code.

The "analytical" approach [1] computes, as a function of the radial position perpendicular to the field lines, the average kinetic energy of the electrons and the average secondary electron yield (SEY). A uniform cloud of secondary electrons, with $2 \mathrm{eV}$ kinetic energy, is kicked by a Gaussian bunch in a dipole field where all the electrons are constrained to move vertically. The integration of the equation of motion allows one to compute all the trajectories of any electrons inside the vacuum chamber. The kinetic energy gain is derived from electron trajectories. Integration of the energy gain during the bunch passage along the vertical field lines yields the average electron energy gain as a function of radial position. This average kinetic energy is used together with laboratory measurements of SEY to estimate the average $\langle\mathrm{SEY}\rangle$ curve, after the bunch passage, as a function of radial position.

Figure 6 shows that only a fractional part of the vacuum chamber surface has a $\langle\mathrm{SEY}\rangle$ above 1 , i.e., a band from 0 to $10 \mathrm{~mm}$ in the present case. Mainly, the secondary electrons located in this volume will participate in the multipacting process. Moreover, since the electrons are vertically constrained by the field lines, the cloud will remain in this area. From Fig. 6, the average kinetic energy of these multipacting electrons incident onto the vacuum chamber is $\sim 50 \mathrm{eV}$. The electron cloud

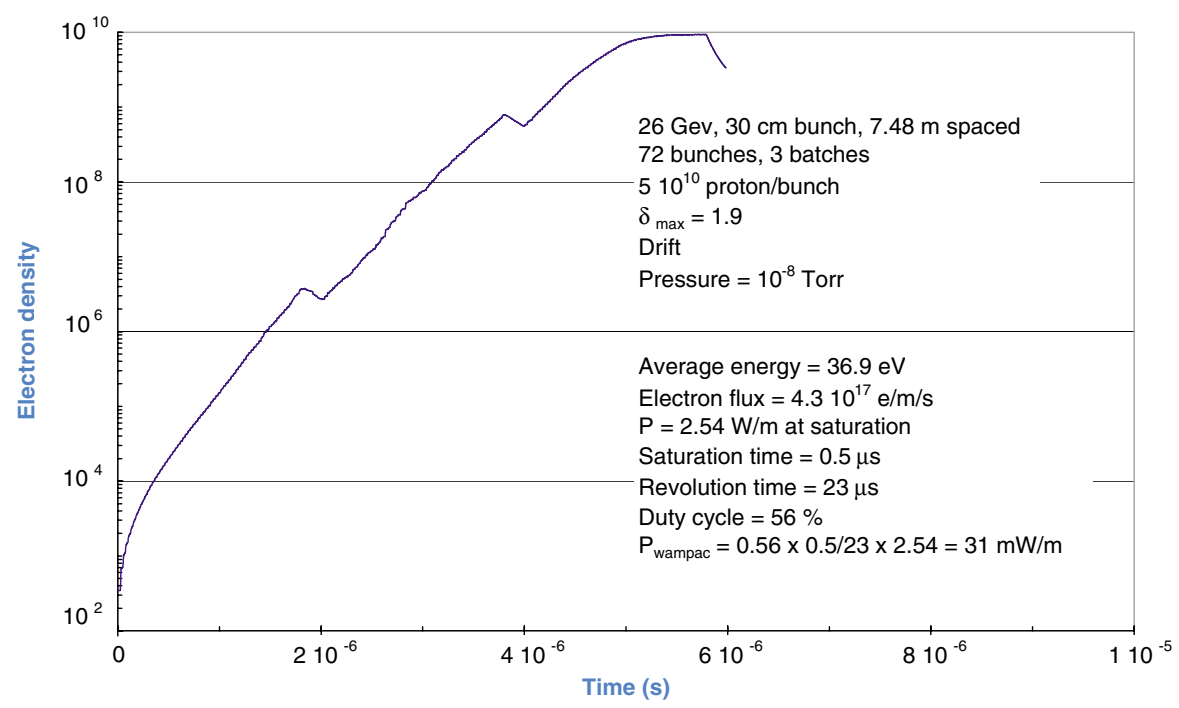

FIG. 7. (Color) Electron density in WAMPAC computed for a maximum SEY of 1.9. 
TABLE III. Simulated power in WAMPAC as a function of the maximum of the SEY.

\begin{tabular}{cccccc}
\hline \hline $\begin{array}{c}\text { Pressure } \\
(\text { Torr })\end{array}$ & $\delta_{\max }$ & $\begin{array}{c}\langle E\rangle \\
(\mathrm{eV})\end{array}$ & $\begin{array}{c}\text { Flux } \\
(e /(\mathrm{m} / \mathrm{s}))\end{array}$ & $\begin{array}{c}\text { Power at } \\
\text { saturation } \\
(\mathrm{W} / \mathrm{m})\end{array}$ & $\begin{array}{c}\text { WAMPAC } \\
\text { Power } \\
(\mathrm{mW} / \mathrm{m})\end{array}$ \\
\hline $10^{-8}$ & 1.90 & 36.9 & $4 \times 10^{17}$ & 2.54 & 31 \\
$10^{-8}$ & 1.95 & 32.9 & $5 \times 10^{17}$ & 2.64 & 66 \\
$10^{-8}$ & 2.00 & 29.2 & $6 \times 10^{17}$ & 2.80 & 78 \\
\hline \hline
\end{tabular}

density in a vacuum chamber is given by its saturation limit defined by the space charge and equals about $10^{9}$ electrons/m [11]. Taking into account the revolution time and the number of bunches, the computed power is then $\sim 70 \mathrm{~mW} / \mathrm{m}$ which is in fairly good agreement with the measurements.

The "macroparticle" approach [12] follows the evolution of macroparticles along the three batches of proton bunches. All fundamental ingredients such as pressure, SEY curve, elastic reflection, and space charge are included. The electrons created by ionization are tracked during the acceleration by the successive bunches and tracked in the drift space. When an electron hits the vacuum chamber wall, depending on its energy and the maximum of the SEY curve, it is either reflected or it creates secondary electrons. Based on the SPS beam and vacuum parameters and in situ measurements of SEY, Fig. 7 shows the computed electron density in the WAMPAC during the passage of the LHC-type beam. The increase of electron density due to the three batches is clearly seen. The electron density saturates only at the end of the last batch. From the average energy of the saturated electron cloud, the electron flux at saturation, the saturation time, and the duty cycle, a power of $31 \mathrm{~mW} / \mathrm{m}$ is estimated.

Table III shows a compilation of several simulations performed with the beam parameters of Sec. IV but without magnetic field for reasons of simplicity. In these simulations, the maximum of the SEY, $\delta_{\max }$, was scanned within a range in agreement with in situ measurements performed in another part of the SPS machine [13]. At saturation, i.e., in the case of a machine full of LHC-type bunches, the typical electron flux is $\sim 5 \times 10^{17} \mathrm{e} /(\mathrm{m} / \mathrm{s})$ and the power $\sim 2.5 \mathrm{~W} / \mathrm{m}$. It is shown that the measurements can be reasonably well obtained. However, a strong sensitivity is noted with the variation of $\delta_{\max }$.

\section{ESTIMATING LHC HEAT LOADS}

The heat load measured with the calorimeter inside the SPS can be scaled to estimate the linear heat load into the LHC. If we assume that the electron cloud activity is nearly independent of the chamber diameter in the range 50 to $140 \mathrm{~mm}$ and of the dipole field in the range 0.5 to $8.5 \mathrm{~T}$, only three corrections should be applied. (1) Since multipacting occurs only in the dipole a correction due to the dipole length, $L$, should be added, (2) the filling factor, $f$, and (3) the duty cycle, $d$, of the SPS should be taken into account. Under these assumptions, the LHC linear heat load, $P_{\mathrm{LHC}}$, could be computed from the WAMPAC measurement, $P_{\text {WAMPAC }}$, by

$$
P_{\mathrm{LHC}}=\frac{1}{L \times f \times d} P_{\mathrm{WAMPAC}} \text {. }
$$

With the parameters from Fig. $4, L=0.7 \mathrm{~m}, f=2 / 11$ (three batch are circulating in the SPS but about one batch is required to trigger the electron cloud [9]), $d=56 \%$, and $P_{\text {WAMPAC }}=30$ to $40 \mathrm{~mW}$, the estimated LHC heat load with $5 \times 10^{10}$ protons $/$ bunch $^{-1}$ in a dipole region and a maximum SEY of about 1.9 is [13]

$$
P_{\mathrm{LHC}} \approx 0.4-0.5 \mathrm{~W} \mathrm{~m}^{-1} \text {. }
$$

\section{CONCLUSIONS}

Preliminary measurements with the SPS calorimeter, WAMPAC, are presented. The calorimeter performance agrees with predictions. It has been demonstrated that a linear heat load of $\sim 20 \mathrm{~mW} \mathrm{~m}^{-1}$ can be measured.

Under a dipole configuration, to reduce the electron cloud activity threshold, a power of 40 to $60 \mathrm{~mW} / \mathrm{m}$ was measured when LHC-type beams were circulating in the SPS. The measurements performed in the SPS are in reasonable agreement with the code predictions. Further investigations are required to validate these codes.

The equivalent LHC linear heat load into the dipole was estimated to be at least $\sim 0.5 \mathrm{~W} \mathrm{~m}^{-1}$ for a current of $5 \times 10^{10}$ protons $/$ bunch $^{-1}$ and a Cu surface having a maximum $\mathrm{SEY}$ of $\sim 1.9$.

To reduce the vertical aperture to $40 \mathrm{~mm}$ and simulate closer the LHC arc beam screen conditions, a new calorimeter has been installed during this shutdown in a SPS dipole chamber. Since predicted vertical electron stripes have been shown to exist [9], this new calorimeter might be equipped, in the future, with a perforated copper liner and allow a direct measurement of the heat load which could be dissipated onto the LHC cold bore.

Finally, the COLDEX, an instrument to simulate as close as possible the arc beam vacuum system, was installed during this shutdown. Comparison of beam induced gas desorption and heat load deposited by a LHCtype beam in a room temperature and in a cryogenic environment shall be performed.

\section{ACKNOWLEDGMENTS}

The work performed, during the installation of WAMPAC in the SPS tunnel, by the LHC-VAC SL section and especially the team of G. Mathis is gratefully acknowledged. We would like to thank G. Arduini, the SL 
operation group, and M. Jimenez for providing and coordinating the LHC-type beams in the SPS. O. Gröbner, G. Rumolo, and F. Zimmermann are acknowledged for allowing the benchmarking of their code.

[1] O. Gröbner, in Proceedings of the Particle Accelerator Conference, PAC'97, Vancouver, Canada, 1997 (LHC Project Report No. 127, 1997).

[2] O. Brüning, LHC Project Report No. 158, 1997.

[3] M. Furman, LHC Project Report No. 180, 1998.

[4] F. Zimmermann, in Proceedings of the XI Chamonix Workshop, 2001 (CERN, Geneva, 2001).

[5] P. Lebrun, LHC Project Report No. 211, 1998.

[6] Th. Durand, http://lhc-mgt-hlwg.web.cern.ch/lhc-mgthlwg/
[7] G. Arduini, K. Cornelis, J. M. Jimenez, G. Moulard, M. Pivi, and K. Weiss, in Proceedings of the $X$ Chamonix Workshop, 2000 (CERN, Geneva, 2000).

[8] B. Jenninger, LHC Project Note No. 189, 1999.

[9] M. Jimenez, LHC Machine Advising Committee, 2001.

[10] M. Jimenez (private communication).

[11] L. Vos, LHC Project Note No. 150, 1998.

[12] G. Rumolo and F. Zimmermann, Phys. Rev. ST Accel. Beams 5, 121002 (2002).

[13] B. Henrist (private communication). The secondary electron yield measurement device is installed in SPS sector 520 where most of the time a dipole field of $70 \mathrm{G}$ is applied. The measurement was performed just before (24/10/01, 0h22) and after (25/10/00, 8h511) the MD period and the SEY did not change appreciably during this period. 